\title{
Hertz Contact Problem Treated by Isogeometric Analysis
}

\author{
Kunpeng HAO ${ }^{1, a}$, Guolai YANG ${ }^{2, b}$ and Qingsi CHENG $^{2, c}$ \\ ${ }^{1}$ Xi'an Kunlun Industry Group Co.Ltd,Xi'an, Province Shanxiu, P.R. China \\ ${ }^{2}$ Nanjing University of Science \& Technology, Nanjing, Province Jiangsu, P.R. China \\ akpsip@sina.com, byyanggl@njust.edu.cn, ${ }^{\mathrm{c}}$ chengqingsi@hotmail.com
}

Keywords: Isogeometric analysis, Hertz Contact, Augmented Method, NURBS.

Abstract. The present penalty-based contact treatment will lead to a simple implementation, and it is difficult to determine a suitable value of the penalty parameter. The usual way is adopting an appropriate multiple of the modulus of elasticity. In this paper, an augmented method for isogeometric contact problem was presented, and this method can alleviate the influence of parameter. As an example, a Hertz contact problem is presented, and the results are compared with Hertz theory.

\section{Introduction}

As one of the most challenging task in computational mechanics, contact problem faces two difficulties in traditional FEA: the surface normal will jump across facet boundaries and geometric inexactness results from facet approximation of contact surface ${ }^{[1]}$. NURBS has two natural advantages on contact problem: preserving geometric continuity and facilitating patch-wise contact search. It has been shown that isogeometric analysis which utilizes NURBS for both geometric description and analysis can circumvent geometry-induced problems on contact analysis.

The usual way to formulate the normal contact pressure as $p_{N}=\varepsilon g_{N}$ ( $g_{N}$ is the gap between the contact surfaces and $\varepsilon$ is the normal penalty parameter). The advantages of the penalty approach are obvious: the technique is quite simple, and is readily interpreted from a physical standpoint. This formulation introduces no additional equations or field variables. Unfortunately, it is also well-known that penalty methods suffer from ill-conditioning that worsens as penalty values are increased, while constraints are satisfied exactly only in the limit of infinite penalty values ${ }^{[2]}$. The augmented technique is considered an alternative approach to circumvent these difficulties.

In this paper, we apply the augmented method on isogeometric contact analysis. The numerical framework follows that in [3]. Here, we no longer simply consider the contact pressure as $p_{N}=\varepsilon g_{N}$, but introduce an augmented parameter to reduce the impact of the choice of normal penalty parameter.

\section{Augmented Treatment of Isogeometric Contact Analysis}

\section{Isogeomtric contact analysis}

Generally, we assume that two bodies in contact are elastic and all the external forces are potential forces. Let $\mathrm{c}=\mathrm{Y}^{(1)} \cap \mathrm{Y}{ }^{(2)}$ denote the contact surface. The total potential is written as:

$$
\Pi=\Pi_{e}+\Pi_{e x t}+\Pi_{c}
$$

Where $\Pi_{e}$ is the elastic energy, $\Pi_{e x t}$ is the potential of external forces, and $\Pi_{c}$ is the contact potential. Here $p$ is the Lagrangian multiplier, and $g_{N}$ is the gap function. They are subjected to the Kuhn-Tucker condition. The penalty regularization replaces the multiplier with the penalty functional:

$$
\Pi_{c}=\frac{1}{2} \int \varepsilon\left\langle-g_{N}\right\rangle^{2} d a
$$


Where <->stands for the Macaulay bracket,. This formulation allows for some small penetration controlled by the parameter $\varepsilon$. The variation of $\Pi_{c}$ gives

$$
\delta \Pi_{c}=\int_{c} \varepsilon g_{N} \delta g_{N} d a=\int_{c} \varepsilon\left[X^{(1)}-X^{(2)}\left(V_{P}\right)\right] \cdot\left(\delta X^{(1)}-X^{(2)}\right) d a
$$

$X^{(1)}$ is the given point ony ${ }^{(1)}$, which can be given on the contact surfaces

$$
X^{(1)}=\sum_{I \in N c r l} N_{1}^{(1)}\left(\mathrm{u}^{1}, \mathrm{u}^{2}\right) q_{1}^{(1)}
$$

$X^{(2)}\left(V_{\mathrm{P}}\right)$ is the unique closet point ony ${ }^{(2)}$, which can be given on the contact surfaces

$$
X^{(2)}=\sum_{I \in \text { Nertl }^{(2)}} N_{1}^{(2)}\left(v^{1}, v^{2}\right) q_{1}^{(2)}
$$

Where $N_{1}^{(i)}$ is NURBS shape function, $q_{1}^{i}$ is control point, and $N c r t l^{(i)}$ represents the set of control points for each body. Thus, we can get:

$$
\delta \Pi_{c}=-\sum_{I \in N_{c r t}(1)} R_{I}^{(1)} \delta q_{I}^{(1)}-\sum_{I \in N_{c r t l}^{(2)}} R_{J}^{(2)} \delta q_{J}^{(2)}
$$

The contact residual vectors are identified:

$$
\begin{aligned}
& R_{I}^{(1)}=\sum_{i \in G^{(1)}} \varepsilon W_{i} J^{(1)}\left(u_{i}\right) N_{I}^{1}\left(u_{i}\right)\left[X^{(2)}\left(V_{p i}\right)-X^{(1)}\left(u_{i}\right)\right] \\
& R_{I}^{(2)}=\sum_{i \in G^{(1)}} \varepsilon W_{i} J^{(1)}\left(u_{i}\right) N_{J}^{2}\left(V_{p i}\right)\left[X^{(1)}\left(u_{i}\right)-X^{(2)}\left(V_{p i}\right)\right]
\end{aligned}
$$

Here $W_{i}$ is the integration weight, $J^{(1)}\left(u_{i}\right)$ is the surface Jacobian ony ${ }^{(1)}, G^{(1)}$ is the set of Gauss points that in contact withy ${ }^{(2)}$. To present the stiffness matrix, we introduce the notations as:

$$
\mathbf{K}=\left[\begin{array}{ll}
K^{(11)} & K^{(12)} \\
K^{(21)} & K^{(22)}
\end{array}\right], \quad K^{(i j)}=\frac{\partial R_{I}^{i}}{\partial q_{J}^{j}}
$$

\section{Augmented treatment}

The concept of the method is remarkable simple, we formulate the contact pressure as:

$$
p_{N}=\varepsilon g_{N}+\lambda_{N}
$$

If we update the $\lambda$ as:

$$
\lambda_{N}^{(k+1)}=\left\langle\lambda_{N}^{(k)}+\varepsilon g_{N}^{(k)}\right\rangle
$$

The gap $g_{N}^{(k)}$ will keep decreasing, and ultimately, the $\lambda_{N}$ will approach the contact pressure $p_{N}$. The following table shows the augmented algorithm for frictionless contact.

Table 1 Augmented algorithm for frictionless contact

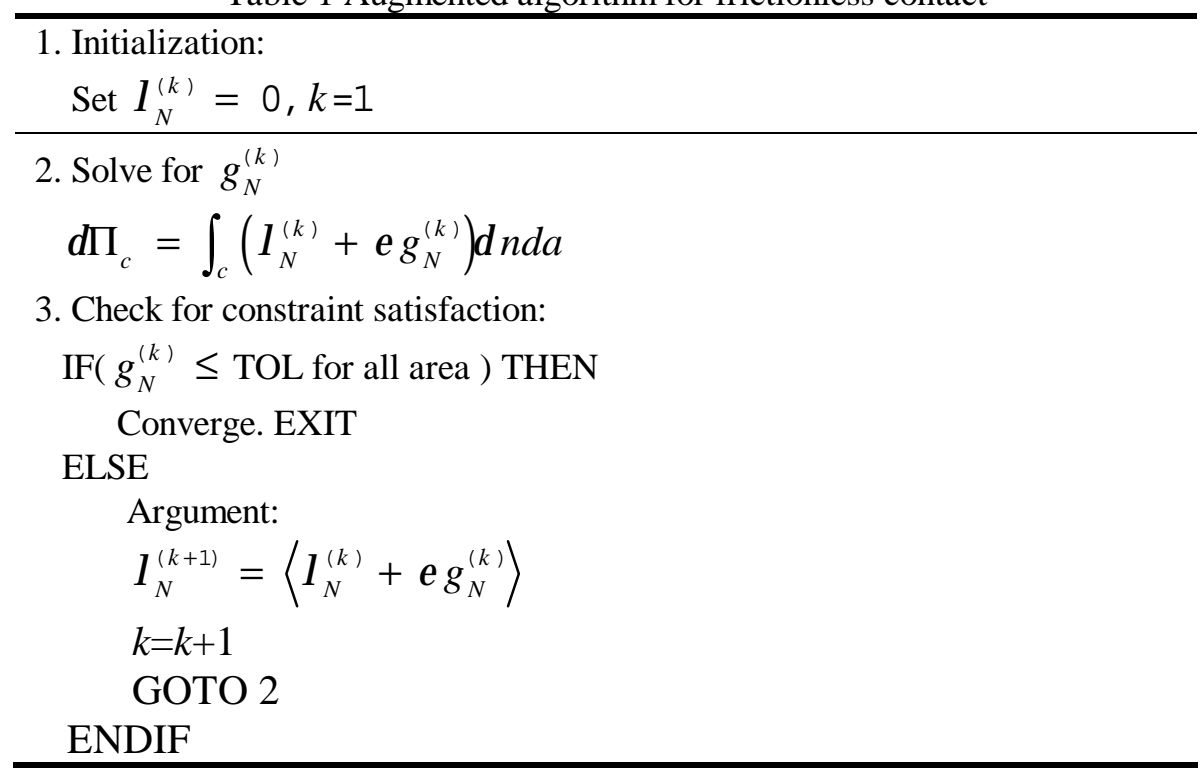




\section{Numeric Simulation and Analysis}

The numerical example is Hertz contact problem, Figure 1 shows the geometry model and the coarsest mesh.
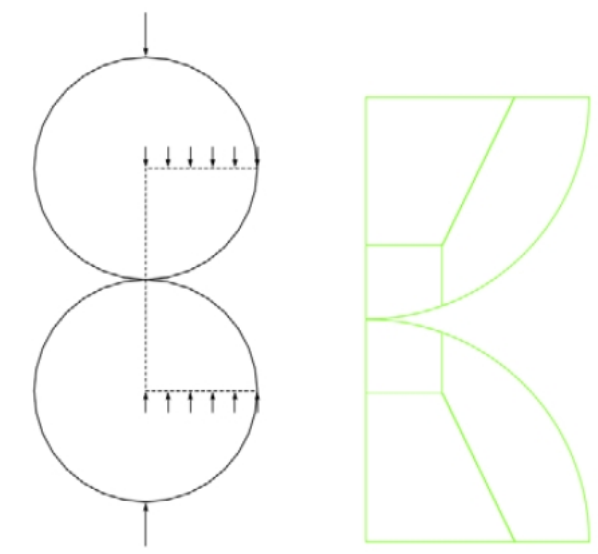

Fig. 1 The geometry model and the coarsest NURBS mesh

Without applying the augmented method, the choice of normal penalty parameter $\varepsilon$ will affect the contact area and also the contact pressure. Figure 2 shows that if $\varepsilon$ is too small $(\varepsilon=10 \mathrm{E}, 20 \mathrm{E}, 50 \mathrm{E}$; E is the modulus of elasticity), the contact area will be larger than theoretical value and cause the contact pressure to be smaller. In this situation, the larger $\varepsilon$ we use, the contact pressure will be closer to the theory value.

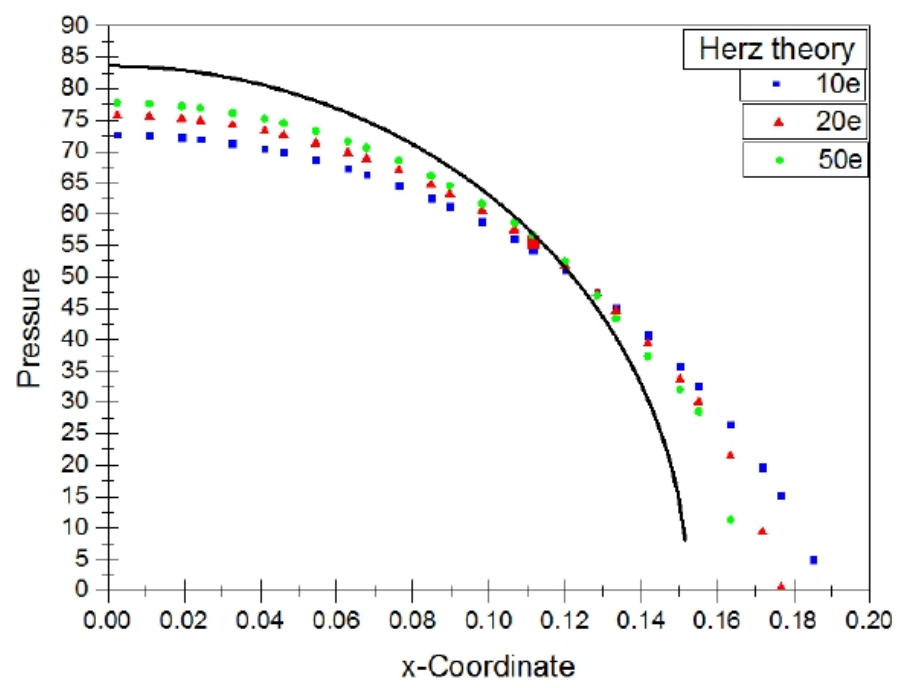

Fig.2 Pressure distributions on the contact surfaces 


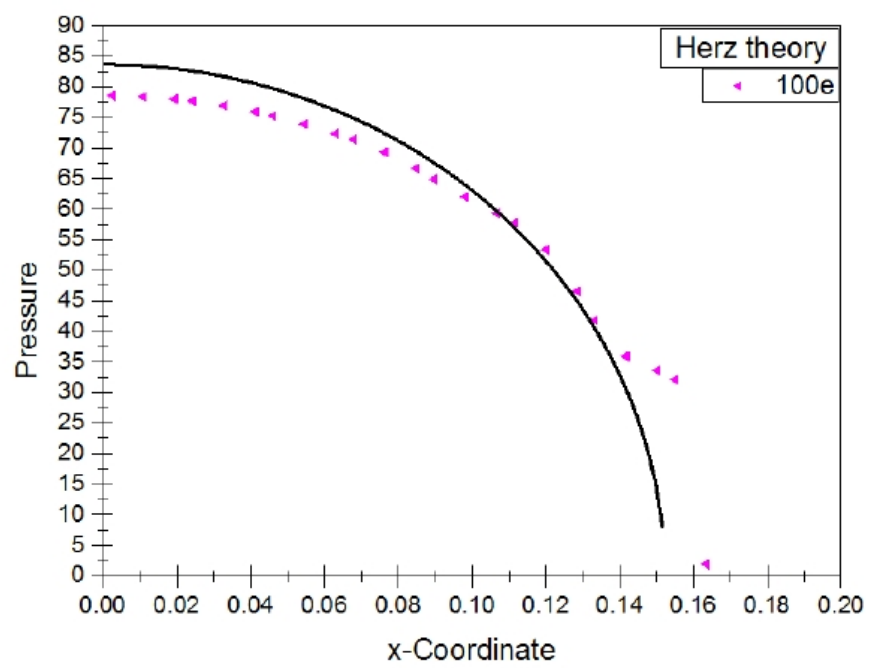

Fig. 3 Pressure distributions on the contact surfaces

However, figure 3 shows that if $\varepsilon$ is too large $(\varepsilon=100 \mathrm{E})$, the contact pressure will be unstable between the contact area and un-contact area.

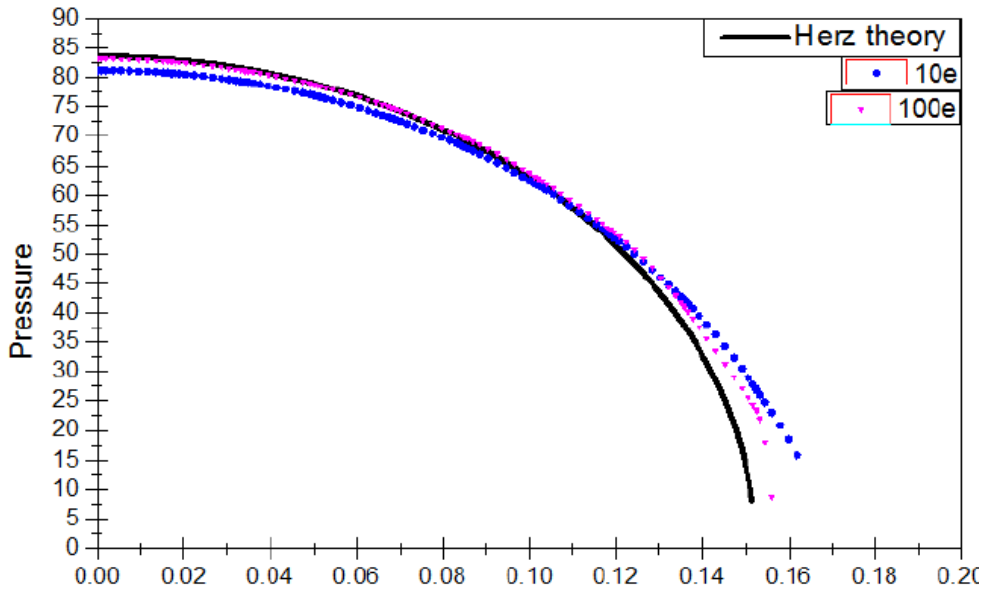

Fig.4 Pressure distributions of augmented treatment

Figure 4 present the results of augmented treatment, it shows that as the $\varepsilon$ grow from $10 \mathrm{E}$ to $100 \mathrm{E}$, the value of contact area and contact pressure are much close than before. This means that the influence of epsilon has been alleviated.

\section{Conclusions}

We have recommended an augmented treatment of isogeometric contact analysis. Numerical examples have demonstrated that the method reduce the impact of the normal penalty parameter $\square$. However, the result in the current study still does not match Hertz solution, and s further study is needed to improve the results. This paper focus on the frictionless contact problem, The extension to frictional contact is the task of an ongoing research.

\section{Acknowledgement}

This work was financially supported by the program of Natural Science Foundation of China (Grant No. 11172139).

\section{Literature References}

[1] T.J.R. Hughes, J.A. Cottrell, Y. Bazilevs. Isogeometric Analysis: CAD, Finite Elements, NURBS, Exact Geometry and Mesh Refinement, Comput. Meth. Appl. Mech. Engrg, 2005,194: 4135-4195. 
[2] J. C. SIMO, T. A. LAURSEN. An Augmented Lagrangian Treatment of Contact Problems Involving Friction. Computers \& Structures, 1992:42:97-116,.

[3] J. LU. Isogeometric Contact Analysis: Geometric Basis and Formulation for Frictionless Contact. Comput. Methods Appl. Mech. Engrg. 2011,200: 726-741.

[4] P. Wriggers, Computational Contact Mechanics, second ed., Springer-Verlag, Berlin Heidelberg, 2006. 